\title{
Structure and equation of state of interaction site models for disc-shaped lamellar colloids
}

\author{
Dino Costa ${ }^{1 *}$, Jean-Pierre Hansen ${ }^{2}$, and Ludger Harnau ${ }^{3,4}$ \\ ${ }^{1}$ Dipartimento di Fisica, Università di Messina Contrada Papardo, C.P. 50, 98166 Messina - Italy \\ ${ }^{2}$ Department of Chemistry, University of Cambridge Lensfield Road, Cambridge CB2 1EW - UK \\ ${ }^{3}$ Max-Planck-Institut für Metallforschung, Heisenbergstr. 3, D-70569 Stuttgart - Germany \\ 4 Institut für Theoretische und Angewandte Physik, \\ Universität Stuttgart, Pfaffenwaldring 57, D-70569 Stuttgart - Germany
}

\begin{abstract}
We apply RISM (Reference Interaction Site Model) and PRISM (polymer-RISM) theories to calculate the site-site pair structure and the osmotic equation of state of suspensions of circular or hexagonal platelets (lamellar colloids) over a range of ratios of the particle diameter over thickness $D / \sigma$. Despite the neglect of edge effects, the simpler PRISM theory yields results in good agreement with the more elaborate RISM calculations, provided the correct form factor, characterizing the intramolecular structure of the platelets, is used. The RISM equation of state is sensitive to the number $n$ of sites used to model the platelets, but saturates when the hard spheres, associated with the interaction sites, nearly touch; the limiting equation of state agrees reasonably well with available simulation data for all densities up to the isotropic-nematic transition. When properly scaled with the second virial coefficient, the equations of state of platelets with different aspect ratios $D / \sigma$ nearly collapse on a single master curve.
\end{abstract}

\section{INTRODUCTION}

Onsager's celebrated theory [1] of suspensions of rodlike or disc-like cylindrical particles of diameter $D$ and length (thickness) $L$ predicts an isotropic to nematic phase transition, both in the rod limit $L / D \gg 1$ and in the plate limit $L / D \ll 1$, on purely entropic grounds. While Onsager's prediction received abundant experimental confirmation for several lyotropic colloidal systems of elongated rod-like particles, like the much studied TMV virus [2], the existence of such a transition in dispersions of lamellar particles, first seen by Langmuir [3], was only recently confirmed by experiments on polymergrafted gibbsite platelets [4]. From a theoretical point of view, there exists no equivalent, in the case of platelets, of the Onsager limit, whereby the equation of state of hard rods is accurately described by the virial series truncated after second order in the limit $L / D \rightarrow \infty$. The contribution of higher order virial coefficients to the equation of state of the cylinders remains large, even in the limit $L / D \rightarrow 0$ (infinitely thin plates), except at very low densities 5]. For platelets of finite thickness $(L / D \neq 0)$, virial coefficients of order higher than two $\left(B_{3}, B_{4}, \ldots\right)$ are not available. Hence there is a clear need to develop accurate theories for simple models of plate-like particles. Attempts have been made along various lines:

- Scaled particle theory [a] , which is very successful for hard sphere fluids, has been extended to prolate or oblate hard ellipsoids of revolution [7], with moderate success when gauged against Monte Carlo simulations [8]. Much less work has been devoted to disc-shaped platelets and the results for infinitely thin platelets $(L / D=0)[9]$ overestimate Monte Carlo data for the equation of state

\footnotetext{
${ }^{*}$ Corresponding author, email: dino.costa@unime.it
}

quite significantly at densities approaching the isotropic to nematic transition 5]. Scaled particle theory, or its extension by Boublik [10], also overestimates the pressure of the closely related model of "cut hard spheres", for which Monte Carlo data are available for several aspect ratios $L / D[11,12]$.

- Onsager theory, based on the second virial coefficient $B_{2}$ alone, can be "rescaled" according to the prescription of Parsons [13] and Lee [14]. Although this semi-empirical procedure gives reasonably good results for hard rod systems $(L / D>1)$, it is much less satisfactory for hard discs $(L / D<1)[15$.

- Rosenfeld's very successful "fundamental measure theory" for hard sphere systems [16] has been extended to the Zwanzig model [17] for square platelets [18], but a direct comparison with disc-shaped particles is not possible because within the Zwanzig model the platelets can only have three discrete orthogonal orientations.

- Instead of modelling platelets as geometrically simple convex bodies (like flat cylinders or cut spheres), an alternative is to consider platelets to be rigid, ordered arrays of interaction sites, distributed over the surface of a disc in some regular pattern [19]. Two examples considered in this paper are shown in Fig. 1 Site-site interactions are assumed to be spherically symmetric, i.e. to depend only on the distance between sites on different platelets. In Ref. 19] the site-site pair potential was chosen to be of the screened Coulomb form, to mimic a dispersion of highly charged Laponite platelets [20] in the presence of added salt, and the pair structure was determined by Molecular Dynamics simulations.

In the present paper we return to this interaction site model, but replace the screened Coulomb interaction by a hard-sphere repulsion, in order to mimic as far as possible suspensions of uncharged, flat, cylindrical platelets. The objective is to calculate the pair structure and osmotic equation of state from RISM (Reference Interaction Site 

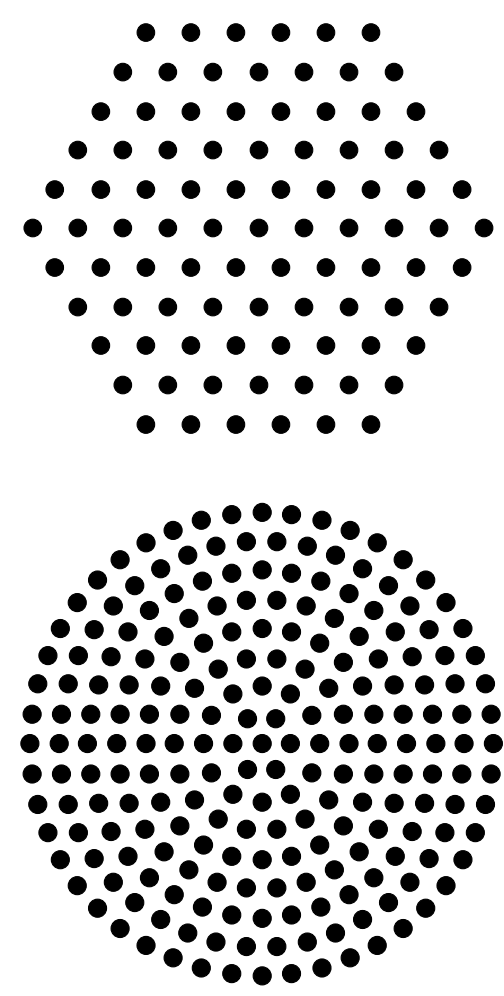

FIG. 1: Schematic representation of site patterns of model platelets investigated in this work.

Model, [21, 22]) and PRISM (polymer-RISM, [23, 24]) integral equations for the site-site pair distribution functions. A systematic comparison will be made between the results from the RISM and PRISM theories, in order to quantify the importance of the pre-averaging procedure and of "edge" effects, which are involved within the simpler PRISM theory, where all sites are assumed to be equivalent 24]. Wherever possible, the theoretical predictions will be compared to available simulation data. Parts of the present work were briefly reported elsewhere [25].

\section{INTERACTION SITE MODELS}

The lamellar systems under consideration contain $N$ disc-shaped platelets of diameter $D=2 R$ inside a volume $V$. Each platelet carries a regular pattern (or grid) of $n$ interaction sites occupied by a hard sphere of diameter $\sigma$. We consider the two grids pictured in Fig. 1] The circular grid is made up of $n_{\mathrm{r}}$ concentric rings of diameter $d_{i}=(i-1)(D-\sigma) /\left(n_{\mathrm{r}}-1\right)$, with $1 \leq i \leq n_{\mathrm{r}}$, comprising each 1 (for $i=1$, i.e. at the origin) or $6(i-1)$ sites, adding up to a total of $n=7,19, \ldots$, 271 sites for $n_{\mathrm{r}}=2,3, \ldots, 10$. The grid has six-fold symmetry around the axis orthogonal to the disc, and along each of the in-plane symmetry axes, hard spheres on neighbouring sites overlap whenever the aspect ratio $D / \sigma<2\left(n_{\mathrm{r}}-1\right)$. For small numbers of rings, it is clear that the resulting geometric object is very different from a convex body, since the interaction-site platelet will have holes, or a highly corrugated surface, depending on the relative values of $D / \sigma$ and $n_{\mathrm{r}}$. For any given $D / \sigma$, the platelet constructed from concentric rings of spheres will tend to a cylinder of the same aspect ratio only in the limit $n_{\mathrm{r}} \rightarrow \infty$.

The hexagonal grid shown in Fig. 1 is identical to that or Ref. 19]. Concentric hexagons replace the rings, and the resulting total numbers of sites are identical to those obtained with the circular pattern. The polygonal shape is reminiscent of that of Gibbsite platelets used in recent experiments at the Van't Hoff Laboratory in Utrecht 4, 26].

The interaction between two platelets is purely steric, and consists of the sum of hard sphere interactions between all the pairs of sites on the platelets, say $A$ and $B$ :

$$
v_{A B}=\sum_{i \in A} \sum_{j \in B} v\left(\left|\mathbf{r}_{i}-\mathbf{r}_{j}\right|\right)
$$

where $\mathbf{r}_{i}$ and $\mathbf{r}_{j}$ are the positions of sites $i$ and $j$, with $1 \leq i, j \leq n$ on platelets $A$ and $B$, while $v(r)$ is the familiar hard sphere "potential":

$$
v(r)=\left\{\begin{array}{cl}
\infty & \text { if } r \leq \sigma \\
0 & \text { if } r>\sigma
\end{array}\right.
$$

This completely defines the interaction site model of platelets which we have investigated. The model is obviously athermal, and its reduced thermodynamic properties will only depend on the platelet density $\rho=N / V$. The much-studied Laponite platelets have typical dimensions $D \simeq 25 \mathrm{~nm}$ and $L$ (identified here with the diameter $\sigma$ of the sphere $\simeq 1 \mathrm{~nm}$, corresponding to an aspect ratio $D / \sigma=25$ [20]. The results which will be presented here are for aspect ratios $5 \leq D / \sigma \leq 50$, as well as for infinitely thin platelets $(\sigma=0)$.

\section{RISM AND PRISM THEORIES}

The pair structure of a fluid of identical particles, each carrying $n$ distinct interaction sites, is characterized by a set of $n(n+1) / 2$ site-site intermolecular pair correlation functions $h_{i j}(r)=g_{i j}(r)-1$. These are related to a set of $n(n+1) / 2$ intermolecular direct correlation functions $c_{i j}(r)$ by the RISM Ornstein-Zernike (OZ) relations [21], which are most conveniently written in matrix form and in Fourier space as:

$$
\mathbf{h}(k)=\mathbf{w}(k) \mathbf{c}(k) \mathbf{w}(k)+\rho \mathbf{w}(k) \mathbf{c}(k) \mathbf{h}(k)
$$

where $\mathbf{h} \equiv\left[h_{i j}(k)\right], \mathbf{c} \equiv\left[c_{i j}(k)\right]$, and $\mathbf{w}$ are $n \times n$ symmetric matrices. The elements of $\mathbf{w} \equiv\left[w_{i j}(k)\right]$ are the 
Fourier transforms of the intramolecular correlation functions. Provided the molecules are rigid, we have explicitly:

$$
w_{i j}(k)=\frac{\sin \left(k L_{i j}\right)}{k L_{i j}},
$$

where $L_{i j}$ is the bond length between sites $i$ and $j$ on the same particle. The RISM-OZ system (3) must be supplemented by a set of closure relations relating the $h_{i j}$ and $c_{i j}$. Since only hard-core interactions are present, the simplest choice is the Percus-Yevick (PY) closure, which combines the exact core condition

$$
h_{i j}(r)=-1, \quad r \leq \sigma
$$

with the approximation

$$
c_{i j}(r)=0, \quad r>\sigma .
$$

The number of independent $h_{i j}$ and $c_{i j}$ may be drastically reduced by obvious symmetry considerations. In the circular platelet model of Fig. 1 all sites on a given ring are equivalent, so that the total number of independent correlation functions is reduced from $n(n+1) / 2$ to $n_{\mathrm{r}}\left(n_{\mathrm{r}}+1\right) / 2$. Following Raineri and Stell [27], we introduce the symmetry-reduced matrix $\mathbf{W}(k)$ of intramolecur correlation functions with elements:

$$
\begin{aligned}
\mathbf{W}_{I J}(k) & =\frac{1}{n_{J}} \sum_{j \in J} w_{i j}(k) \\
& =\frac{1}{n_{I}} \sum_{i \in I} w_{i j}(k)=\mathbf{W}_{J I}(k)
\end{aligned}
$$

where the indices $I$ and $J$ run over the number of inequivalent classes of interaction sites and $n_{I}$ or $n_{J}$ are the numbers of equivalent sites of classes $I$ or $J$ in the molecule. We also define the following direct correlation function matrix $\mathbf{C}$ with elements:

$$
C_{I J}(k)=n_{I} \cdot c_{i j}(k) \cdot n_{J}
$$

where $c_{i j}(k)$ is any of the identical direct correlation function of the original $n \times n$ matrix, with $i \in I$ and $j \in J$. The reduced RISM-OZ equation now reads in $n_{\mathrm{r}} \times n_{\mathrm{r}}$ matrix form:

$$
\mathbf{h}(k)=\mathbf{W}(k) \mathbf{C}(k) \mathbf{W}(k)+\rho \mathbf{W}(k) \mathbf{C}(k) \mathbf{h}(k),
$$

which is once more supplemented by the PY closure relations (5) and (6). In the case of the hexagonal grid in Fig. 11 the reduction procedure due to symmetry is slightly more elaborate. Consequently, we made the simple (but slightly inaccurate) assumption that all sites on a given hexagonal shell (also referred to as "ring") are equivalent, which is strictly true only for the $n_{\mathrm{r}}=2$ shell.

Despite its diagrammatic inconsistency [28], RISM has proved to be a successful theory of the pair structure of many simple molecular fluids 22]. In the case of macromolecular and colloidal systems, with very large numbers of interaction sites, the number of coupled RISM equations become intractable, and a considerable simplification follows from the assumption that all interaction sites are equivalent. This leads to the PRISM theory, first applied by Schweizer and Curro to long flexible polymers 23]. PRISM theory neglects end effects in that case. In the case of rigid lamellar particles of finite diameter, the PRISM assumption is clearly less justified, particularly so as the number $n_{\mathrm{r}}$ of concentric rings of sites increases. The set of correlation functions in Eq. (3) is replaced by a single, platelet-averaged function $h$, defined by:

$$
h(k)=\frac{1}{n^{2}} \sum_{i, j} h_{i j}(k)
$$

and similarily for $c(k)$. The platelet-averaged intramolecular correlation function is defined by:

$$
w(k)=\frac{1}{n} \sum_{i, j} w_{i j}(k),
$$

and the resulting single PRISM-OZ relation reads

$$
h(k)=w(k) c(k)[w(k)+\rho n h(k)],
$$

where $\rho n$ is the number density of interaction sites. Note that $w(k)$ is proportional to the intramolecular form factor $F(k)$ of a single platelet:

$$
\begin{aligned}
F(k) & =\frac{1}{n} w(k)=\frac{1}{n^{2}} \sum_{i, j} w_{i j}(k) \\
& =\frac{1}{n}+\frac{1}{n^{2}} \sum_{i \neq j} w_{i j}(k) .
\end{aligned}
$$

which characterizes the geometry of the distribution of sites, and hence the geometric shape of the model platelets. While the form factor accounts for the interference of radiation scattered from different parts of the same particle in an X-ray or neutron diffraction experiment, the local order in the fluid or suspension is characterized by the set of pair distribution functions $g_{i j}(r)$, or, approximately, by the pre-averaged single combination (12). An important particular case is the centre-tocentre pair distribution function $g_{\mathrm{cc}}(r)$. The experimentally accessible total structure factor is:

$$
S(k)=F(k)+\frac{1}{n^{2}} \sum_{i, j} S_{i j}(k) \equiv F(k)+S_{\text {inter }}(k),
$$

where the site-site partial structure factors $S_{i j}(k)$ are the Fourier transforms of the $g_{i j}(r)$. The structure factor provides a direct link with thermodynamics, via the compressibility equation [29]:

$$
\lim _{k \rightarrow 0} S(k)=\rho k_{\mathrm{B}} T \chi_{T},
$$

where $\chi_{T}$ is the isothermal compressibility. The osmotic pressure then follows from:

$$
\beta P \equiv \frac{P}{k_{\mathrm{B}} T}=\int_{0}^{\rho} d \rho^{\prime}[S(k=0)]^{-1}
$$




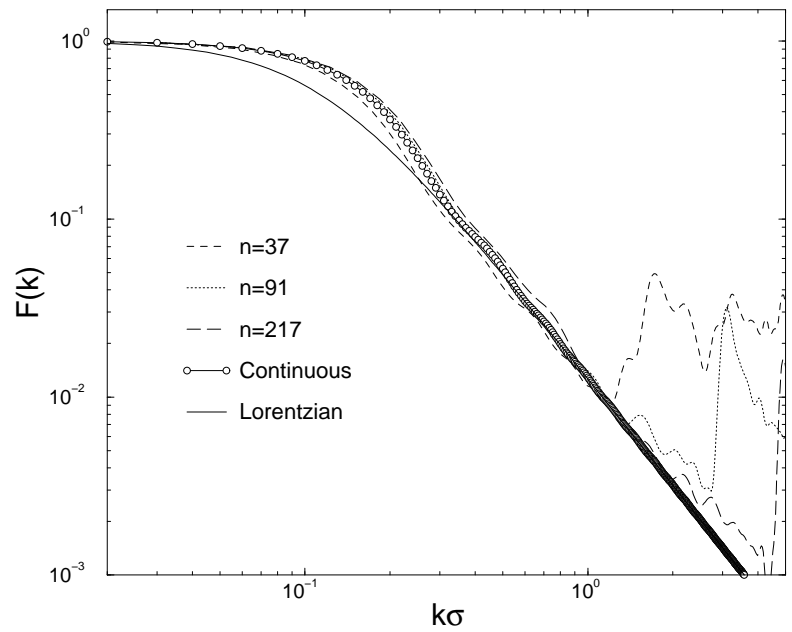

FIG. 2: Form factor of model platelets, Eq. (13), with $D / \sigma=25$ and different numbers $n$ of sites, compared with the continuous distribution limit (17) and the Lorentzian approximant (18). Symbols are defined in the figure.

Returning to PRISM theory, the OZ relation (12) must be supplemented by a closure relation for the preaveraged functions $h$ or $c$. In this work we use throughout the PY closure (5) and (6), both within the RISM and PRISM representations. PRISM theory has so far been mostly applied to polymer solutions and melts, whence the name 24]. For flexible polymers the form factor $F(k)$ follows from a statistical average over single polymer conformations, while in the case of rigid rods or platelets considered in the present work, $F(k)$, or equivalently $w(k)$, are trivially known functions of the wave number $k$. The case of rigid rods was investigated within the PRISM formalism both as the limit of polymers with a persistence length exceeding the overall polymer length [30], and by using the correct form factor (see [31] and references therein).

\section{PAIR STRUCTURE}

We have solved the RISM and PRISM equations for various interaction site distributions, with number of sites $n$ ranging from $n=37\left(n_{\mathrm{r}}=4\right)$ to $n=271$ $\left(n_{\mathrm{r}}=10\right)$, for aspect ratios $D / \sigma$ ranging from 5 to 25 and in the limit $\sigma=0$. Test calculations with $D / \sigma=50$ and $n_{\mathrm{r}}=18$ have also been carried out.

The advantage of using symmetry-reduced RISM and PRISM theories is that $n$ may be increased within a reasonable computational effort, in order to mimic, as accurately as possible, a uniform distribution over the platelet area, and hence a smooth surface of a thin cylinder. In fact, the limit of a continuous distribution may be achieved straightforwardly in the PRISM formalism.

The importance of "holes" between sites on the grid may be seen in Fig. 2] where the form factors $F(k)$, de-
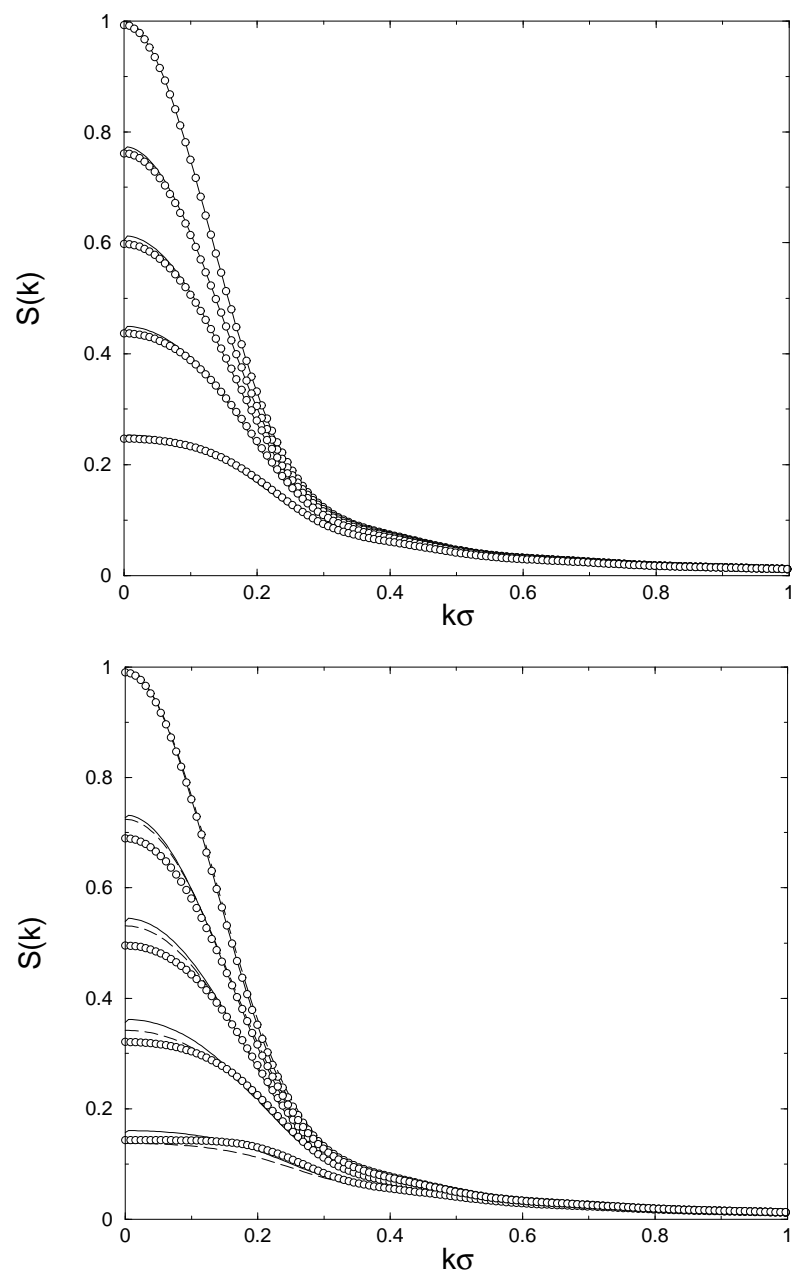

FIG. 3: Total structure factor for model platelets with $D / \sigma=$ 25, and 91 (top) or 217 (bottom) sites. Circles: RISM; lines: PRISM results with the discrete [Eq. (13), full lines], or the continuous [Eq. (17), dashed lines in the bottom panel] form factors. Reduced densities are $\rho^{*}=\rho D^{3}=0.01,0.40,0.80$, 1.40, 2.80 (from top to bottom).

fined in Eq. (13), are plotted for several values of the number of sites, and compared to the limit of a continuos distribution of sites, when the sum in Eq. (13) goes over to an integral on the surface of the platelet, with the result [32]:

$$
F(k)=\frac{2}{(k R)^{2}}\left[1-\frac{J_{1}(2 k R)}{k R}\right],
$$

where $J_{1}$ denotes the cylindrical Bessel function of order one. The discrete nature of the site distribution is reflected in the irregular oscillations visible for $k \sigma>1$ in Fig. 2 In this regime, the correct $k^{-2}$ power law decay at large $k$ is progressively recovered by increasing the number $n$ of sites for a given diameter $D$. A model with $n_{\mathrm{r}}=9$ ( $n=217$ sites $)$, where the distance between nearest-neighbour sites is $1.5 \sigma$, reproduces the continuous form factor (17) with reasonable accuracy. Figure 2 


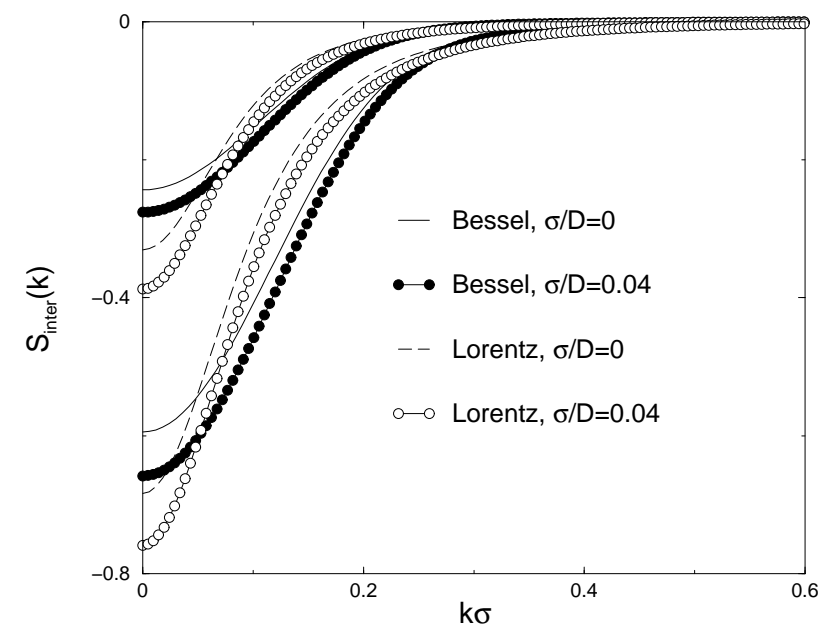

FIG. 4: Intermolecular structure factor $S_{\text {inter }}(k)$, for model platelets with $D / \sigma=25$ or $\infty$, calculated within PRISM, with the Lorentzian form factor [Eq. (18)], and the Bessel form factor [Eq. (17)]. Reduced densities are $\rho^{*}=\rho D^{3}=0.40$ (upper curves) and 1.40 (lower curves).

also displays a simple Lorentzian approximation of the form factor, suitable for analytical calculation [25, 33]:

$$
F(k)=\frac{2}{2+(k R)^{2}} .
$$

Results for the overall structure factor (14), and for the centre-to-centre structure factor obtained by solving the RISM and PRISM equations for the circular pattern of sites shown in Fig. 1 1 are presented in Figures 3 . 5 For the hexagonal pattern, the full and approximate symmetry-reduced RISM calculations yield practically indistinguishable results (not shown), and the structure factors are very similar to those obtained with the circular pattern, for the same number of sites $n$, aspect ratio $D / \sigma$, and reduced density. Figure 3 compares the RISM and PRISM results for the total structure factor at five different densities and for an aspect ratio $D / \sigma=25$. The same discrete form factor defined by Eqs. (4) and (13) was used in the two integral equations for the models with $n=91$ and 217 sites. In the $n=91$ case, RISM and PRISM results are virtually indistinguishable, and this agreement is even better for smaller $n$. The agreement deteriorates somewhat as $n$ increases, as illustrated by the case with $n=217$, showing that the PRISM preaveraging assumption gradually breaks down when the number of sites increases. As visible from Fig. 3, the PRISM structure factors are slightly modified by going to the continuum limit, characterized by the form factor (17), and move closer to the RISM data, pointing to some degree of cancellation of errors.

A comparison of the intermolecular part of the structure factors $S_{\text {inter }}(k)$ [see Eq. (14)], calculated within PRISM in the limit of a continuous distribution of sites $(n \rightarrow \infty)$ using the exact [Eq. [17]], and the approxi- mate [Eq. [18] ], form factors is made in Fig. 4 for size ratios $\sigma / D=0$ and 0.04 ; the results are seen to be very sensitive to the choice of form factor, particularly at small wavenumbers $k$, which will have implications for the equation of state calculated via Eqs. (15) and (16).

Note that the only justification for the use of the approximate Lorentzian form factor is that it allows an analytic solution of the PRISM equation in the limit of infinitely thin $(\sigma / D \rightarrow 0)$ hard as well as charged platelets [25]. Indeed, in that limit the PRISM equation (12), supplemented by the closure relation:

$$
c(\mathbf{r})=c_{0} \delta(\mathbf{r})
$$

can then be solved analytically [33]; the parameter $c_{0}$ is determined by enforcing the exact core condition $h(\mathbf{r}=$ $0)=-1$. The resulting structure factor reads:

$$
\begin{aligned}
S(k) & =w(k)+\rho h(k) \\
& =2\left\{2+2 \rho A(R)+(k R)^{2}\right\}^{-1} \\
A(R) & =8 \pi\left(\frac{R}{\sqrt{2}}\right)^{3}+16 \pi^{2} \rho\left(\frac{R}{\sqrt{2}}\right)^{6} .
\end{aligned}
$$

The result (20) is, not surprisingly, identical to that obtained from the PRISM solution of the thread model, using the same closure (19) and the same approximant (18) to the form factor, with the radius $R$ of the platelet replaced by the radius of gyration $R_{\mathrm{g}}$ of the polymer [33]. This coincidence illustrates the importance of using the correct form factor of the objects under consideration. The form factor $F(k)$ for the platelets is known exactly [cfr Eq. [17)], but only approximately in the case of polymers, where it is often taken to be of the Debye form, valid for ideal (gaussian) polymer coils 34].

RISM results for the centre-to-centre structure factor $S_{\text {cc }}(k)$, calculated for the $n=217$ sites model, are shown in Fig. 5 for two size ratios, $D / \sigma=10$ and 25, and four densities. As expected, the $S_{\mathrm{cc}}(k)$ for the smaller size ratio show more structure because the corresponding effective packing fractions are larger. The corresponding centre-to-centre pair distribution function, $g_{\mathrm{cc}}(r)$, for $D / \sigma=25$, is shown in Fig. 6] and compared to Eppenga and Frenkel's Monte Carlo (MC) data for infinitely thin platelets 5]. The oscillations in the RISM results at short distances reflect the surface "roughness" due to the discrete site distribution, and are of course absent in the $\mathrm{MC}$ data. Bearing in mind the differences between the two models, the agreement between theory and simulation is reasonably good, and all curves exhibit the expected cusp at $r \simeq D$. At the highest density the MC data exhibit a steep rise followed by a striking inflection point around $r \simeq 0.4 D$, which is only faintly reproduced by RISM; for $r \lesssim 0.4 D$, the MC $g_{\mathrm{cc}}(r)$ lies well above the RISM prediction, pointing to some degree of local orientational ordering, which may be regarded as a precursor to the isotropic-nematic transition taking place at $\rho D^{3} \simeq 4[5]$. Pair distribution functions $g_{\mathrm{cc}}(r)$ for the 

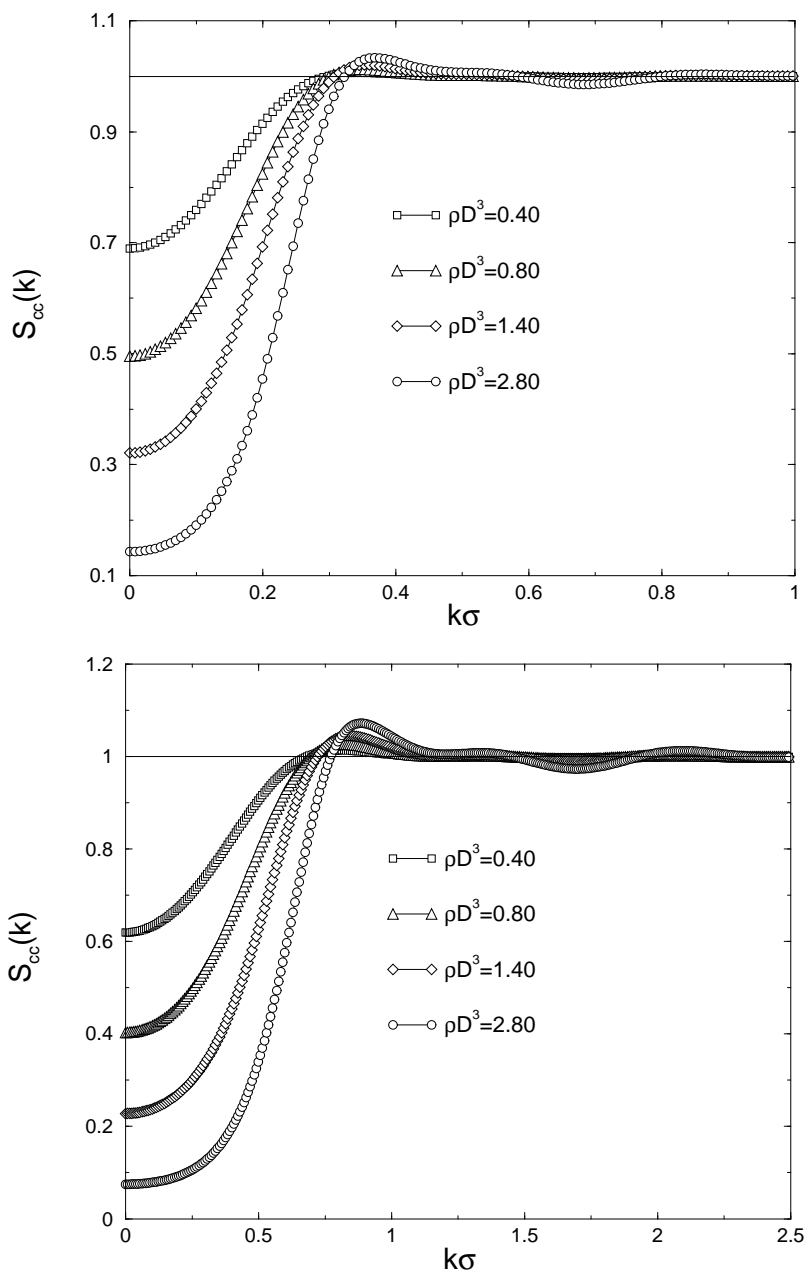

FIG. 5: RISM centre-to-centre structure factors $S_{\mathrm{cc}}(k)$, for platelet models with $D / \sigma=25$ (top) and 10 (bottom), for densities specified by the symbols.

size ratio $D / \sigma=10$ are reported in the bottom panel of Fig. [6 for four densities. The qualitative behaviour is similar to that predicted by RISM for the larger size ratio; a similar cusp appears at $r \simeq D$.

\section{THE EQUATION OF STATE}

From a knowledge of the site-site correlation functions, the only direct route to the osmotic equation of state is via Eqs. (15) and (16). Results from RISM and PRISM calculations for circular site patterns with $37 \leq n \leq 271$ sites, and an aspect ratio $D / \sigma=25$ are shown in Fig. 7 and compared to the MC data for infinitely thin platelets [5]. For any given reduced density $\rho^{*}=\rho D^{3}$, the pressure increases with the number $n$ of sites. This is easily understood, because for lower values of $n$, the distance between neighbouring sites exceeds $2 \sigma$, so that two platelets can, in fact, partially interpenetrate
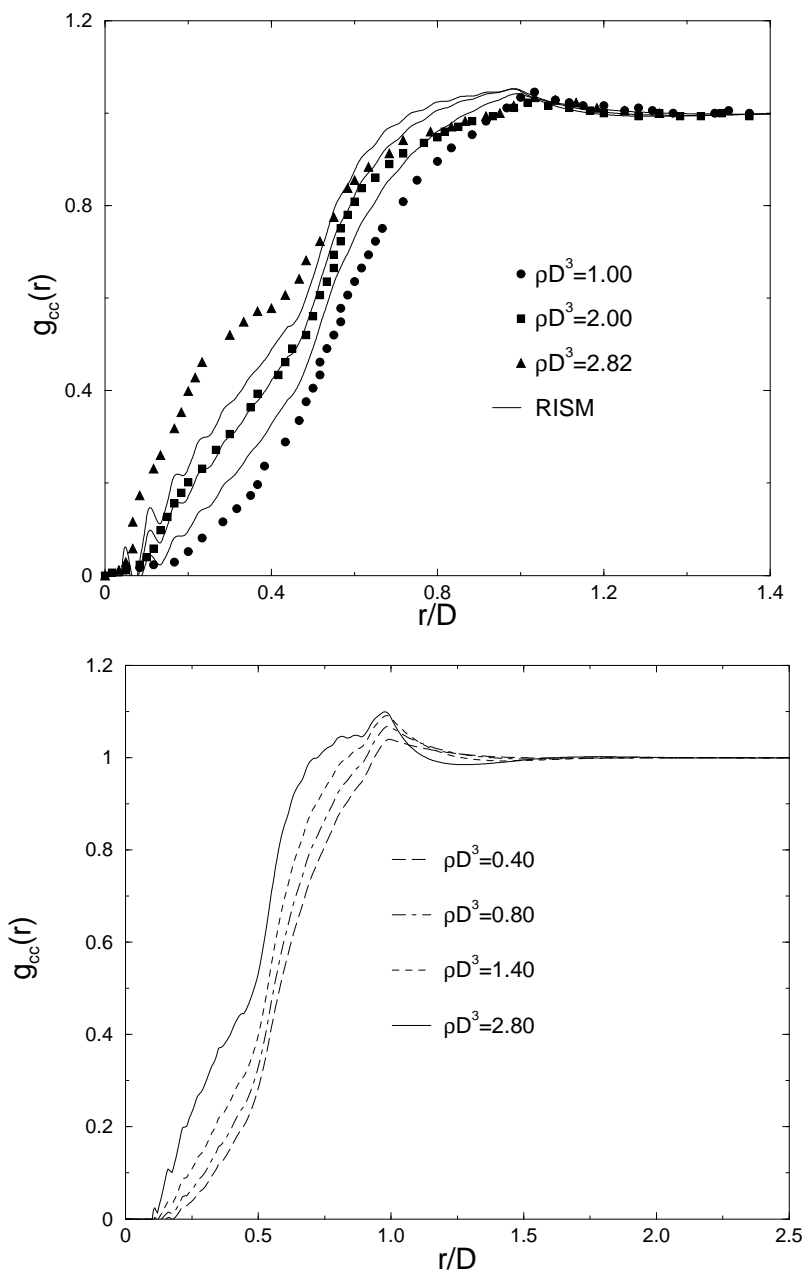

FIG. 6: Top: RISM centre-to-centre radial distribution function for platelets with $D / \sigma=25$ (lines, in order of increasing densities, from left to right). Symbols are the corresponding simulation data for infinitely thin hard discs [5]. Bottom: RISM radial distribution functions for platelets with $D / \sigma=10$.

for favourable relative orientations. But for $n=217$, the distance between neighbouring spheres $(\simeq 1.5 \sigma)$ is small enough to avoid interpenetration, and the value of the pressure saturates, as was checked by RISM test calculations with ten concentric rings, corresponding to $n=271$ sites. These saturated values of the pressure lie about $10 \%$ below the $\mathrm{MC}$ data in the intermediate density range. Interestingly, the MC data show very little curvature of the equation of state for $\rho^{*} \gtrsim 1.5$, and fall below the predictions of RISM and of the fifth order virial expansion at higher densities. This "softening" of the equation of state may be indicative of local orientational ordering prior to the isotropic-nematic transition which occurs at $\rho^{*} \simeq 4$. Such local ordering, already apparent in the pair distribution function shown in Fig. [6] cannot be accounted for by RISM theory, which assumes full isotropy of the local correlations. The PRISM results, ob- 


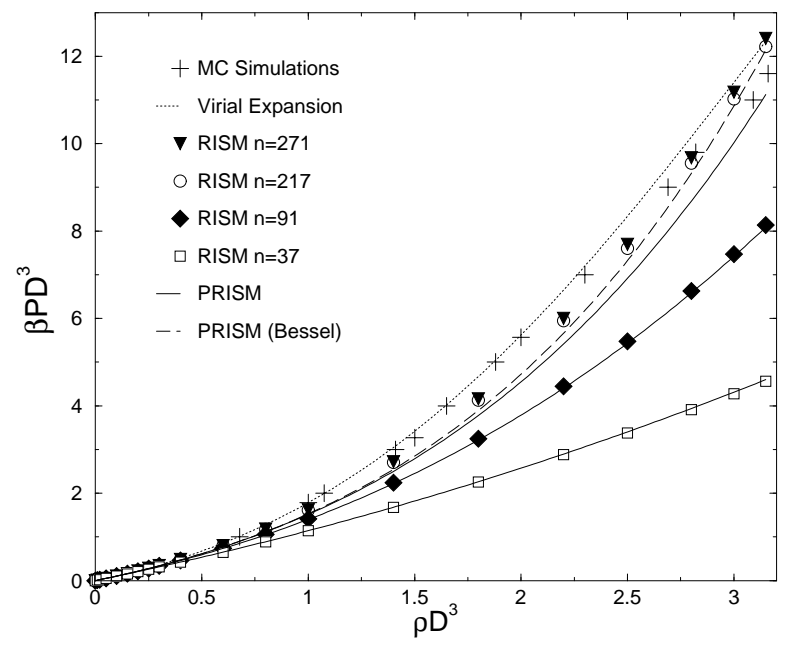

FIG. 7: Equation of state of hard platelets with $D / \sigma=25$ in the isotropic fluid range, as obtained through RISM (symbols) and PRISM (full lines) calculations with $n=37,91$, and 217 sites and the same form factor of Eq. (13). RISM test calculations with $n=271$, and PRISM results with the continuous Bessel form factor, Eq. (17), are also reported. Monte Carlo data and the 5 th order virial expansion for infinitely thin hard platelets are taken form Ref. [5].

tained with the discrete form factor (11) appropriate for a given number of sites, are in excellent agreement with the RISM pressures up to $n=91$. Deviations between PRISM and RISM results become apparent for $n=127$ (not shown) and increasingly so for $n=217$. However, if the continuum limit $(n \rightarrow \infty)$ is taken within PRISM, the form factor goes over to that given in Eq. (17), and the resulting equation of state agrees then closely with the RISM results for $n=217$, which are close to saturation, i.e. to the expected continuum limit within RISM.

Similar RISM and PRISM results for the equation of state of discs with an aspect ratio $D / \sigma=10$ are compared in Fig. 8 to MC data for "cut hard spheres" with the same aspect ratio [11]. The trends are qualitatively the same as for the previous case with $D / \sigma=25$. The "softening" of the "exact" equation of state (as obtained by $\mathrm{MC}$ simulations) is again very striking at packing fractions $\eta \gtrsim 0.2$, where the $\mathrm{MC}$ data are bracketed by the predictions of the 5th order virial expansion (which lie above) and the RISM predictions. Close to the expected isotropic-nematic transition at $\eta \simeq 0.3[1]$ the "saturated" RISM pressure (estimated from calculation for the $n=217$ sites model) nearly coincides with the MC value. The continuum PRISM equation of state is close to the "saturated" RISM result as in the case with $D / \sigma=25$.

The PRISM pressures for $D / \sigma=5,10,15,20,25,50$ and in the limit $\sigma \rightarrow 0$ are collected in Fig. 9, where the platelet density and the ratio $P / k_{\mathrm{B}} T$ are both reduced by the second virial coefficient in the isotropic phase [1]:

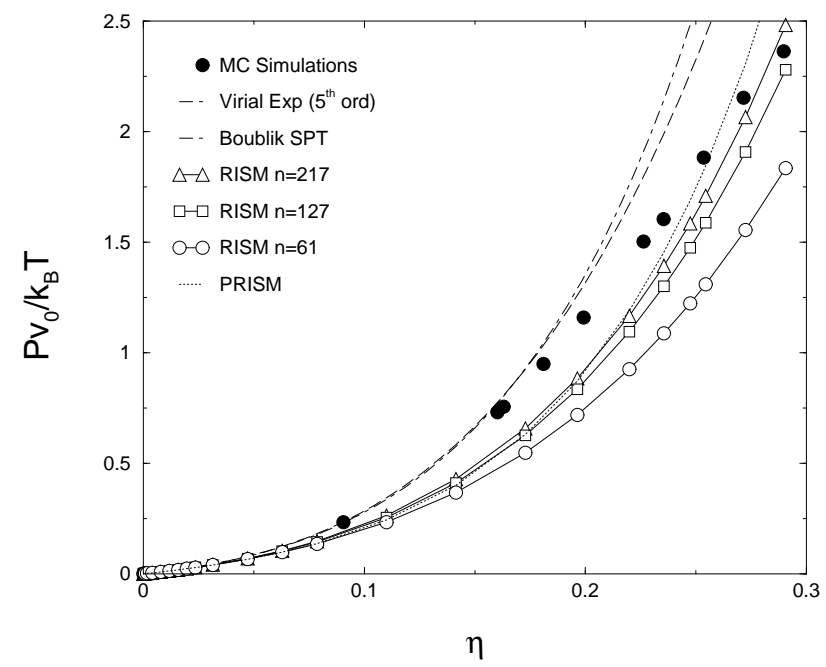

FIG. 8: RISM and PRISM (with Bessel form factor) predictions for the equation of state of model platelets with $D / \sigma=10$. Results are compared with Monte Carlo simulations of cut hard spheres of Ref. [11].

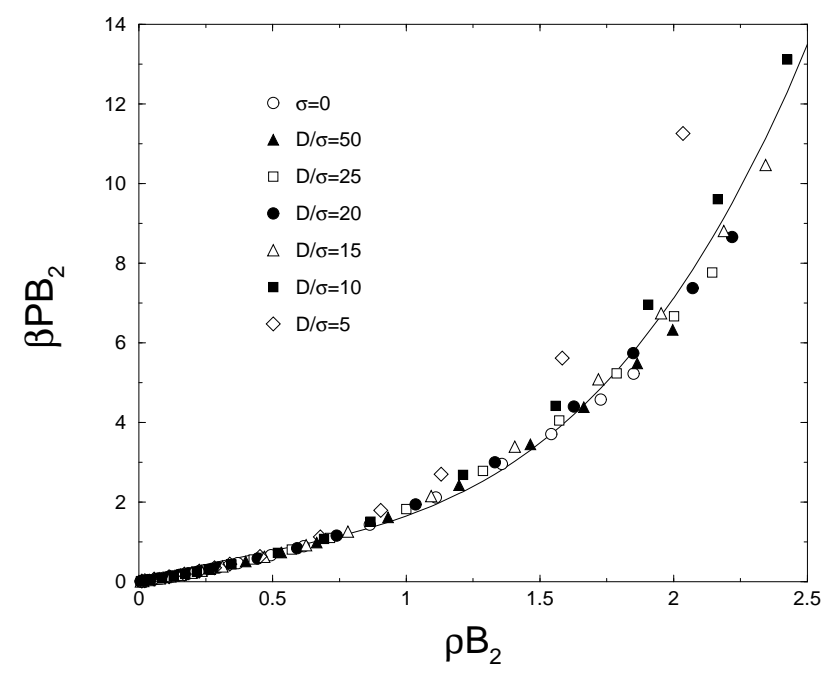

FIG. 9: Equation of state of model platelets in reduced units of pressure and density, $P^{*}=\beta P B_{2}, \rho^{*}=\rho B_{2}$ (where $B_{2}$ is the second virial coefficient). Full line is a cubic fit of all data in the range $10 \leq D / \sigma<\infty$.

$$
B_{2}=\frac{\pi^{2}}{16} D^{3}+\frac{\pi}{8}(3+\pi) D^{2} \sigma+\frac{\pi}{4} D \sigma^{2} .
$$

When $P^{*}=\beta P B_{2}$ is plotted versus $\rho^{*}=\rho B_{2}$, the data nearly collapse on a single "master curve", which is reasonably well fitted by a cubic polynomial. Only the results for $D / \sigma=5$ lie significantly above the master curve for $\rho^{*}>1$.

Equations of state obtained with the circular and the hexagonal grids of Fig. 10 are reported in Fig. 10 for 


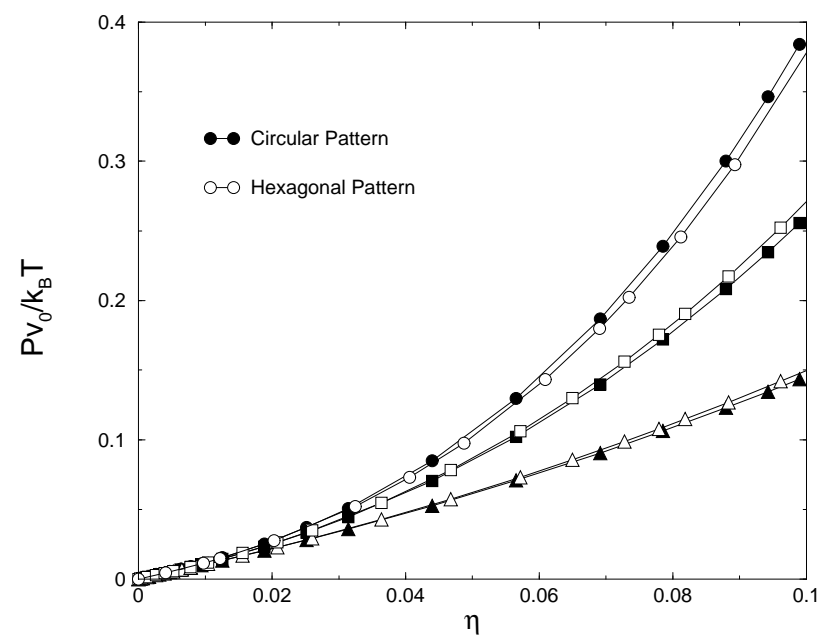

FIG. 10: RISM equation of state for model platelets with different geometries, $D / \sigma=25$ and $n=217$, 91, or 37 sites (from top to bottom). Full symbols: circular geometry; open symbols: hexagonal geometry (see Fig. 1). $\eta=\rho v_{0}$ is the dimensionless packing fraction and $v_{0}$ is the particle volume.

$D / \sigma=25$, and $n=37,91$, and 217 sites. As visible, the pressures of both models agree closely, if the densities $\rho$ are properly reduced by the volume of a circular or hexagonal platelet in the continuum $(n \rightarrow \infty)$ limit.

We finally return to the prediction of the continuum PRISM theory, using the PY closure for finite thickness $D / \sigma=25$ and 50 , and the closure (19) for infinitely thin $(\sigma \rightarrow 0)$ platelets. PRISM results, obtained with the exact (17) and the approximate (18) form factors are reported in Fig. [1] and compared to the RISM predictions for $D / \sigma=25$ and 50 (with $n_{\mathrm{r}}=9$ and 18 , respectively). Two main conclusions which may be drawn from the comparison are the following. First, the pressures are higher when $\sigma=0.04 D$, compared to $\sigma=0.02 D$ (and $\sigma=0$ within PRISM), as expected for obvious excluded volume reasons, but the effect appears to be exagerated by PRISM theory. Finally, as already evident from the comparison of the PRISM structure factors shown in Fig. [4 the form factor has a considerable influence on the PRISM results with the Lorentzian approximant to the exact form factor always leading to an overestimation of the pressure. In view of the present discussion, the good agreement between the predictions of the continuum PRISM theory, obtained with the Lorentzian form factor (18), and the "exact" MC data for infinitely thin platelets [5] noted in Ref. 25], must be considered as partly accidental, due to a cancellation of errors arising from the combination of an inadequate form factor and the assumption of equivalence of all sites.

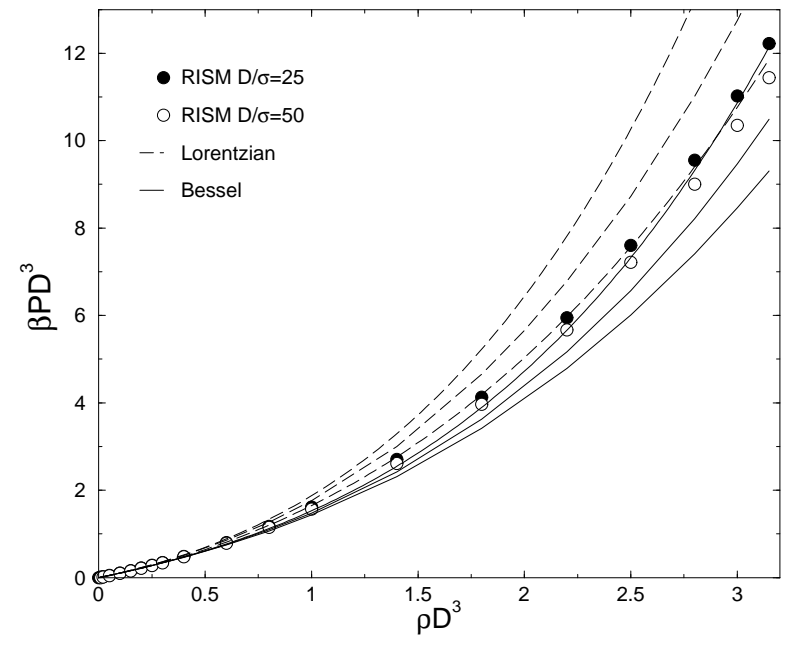

FIG. 11: Equation of state of thin model platelets. RISM (full circles: $D / \sigma=25$, open circles: $D / \sigma=50)$; PRISM with the Lorentzian form factor, Eq. (18) (dashed lines, $D / \sigma=25,50$ and $\infty$, from top to bottom); PRISM with the exact Bessel form factor, Eq. (17) (full lines, $D / \sigma=25,50$ and $\infty$, from top to bottom).

\section{CONCLUSIONS}

We have presented results of what we believe to be the first systematic application of RISM and PRISM theories to rigid, lamellar colloidal particles. While RISM has previously been almost exclusively applied to rigid molecules 22], and PRISM, as indicated by the first letter of the acronym, has been mostly applied to polymers [24], our investigation presents a systematic comparison of the predictions of the two (related) theories as applied to colloidal platelets. In this paper we have restricted our attention to systems with excluded volume (hard core) interactions only between the sites distributed in a circular or hexagonal pattern. For low numbers $n$ of sites, the model platelets have many "holes" or exhibit highly corrugated surfaces, but for sufficiently large $n$, the spheres associated with neighbouring sites touch or overlap, and in the continuum limit $n \rightarrow \infty$, which can easily be dealt with within the PRISM theory, the topology of the multisite plates goes over to smooth cylindrical or hexagonal bodies.

The main findings and conclusions of the present work may be summarized as follows:

(a) RISM theory predicts reasonable pair structure, as embodied in the centre-to-centre pair distribution function (cfr Fig. 6) or the total structure factor (cfr Fig. 3), compared to available simulation data. The fully isotropic form factor used in all our calculations, and the ensuing complete decorrelation between plate orientations and the site-site correlation functions, do not allow a proper description of the local orientational ordering observed in the MC simulations.

(b) The RISM equation of state converges rather slowly 
to the hard platelet limit, as the number of interaction sites increases, but "saturation" is reached when the spheres associated with neighbouring sites are close enough to prevent the interpenetration of platelets. The limiting equation of state is strongly convex and shows no sign of the "softening" observed in MC simulations well before the isotropic-to-nematic transition is reached. The RISM pressures lie systematically below the predictions of a five-term virial series, and of the closely related Boublik equation of state.

(c) The properly scaled RISM equation of state data over a wide range of aspect ratios $D / \sigma$ collapse onto a single "master curve".

(d) The results of the much simpler PRISM theory, which assumes all interaction sites to be equivalent, agree unexpectedly well with their RISM counterparts, when the appropriate form factors are used. In other words, pre-averaging effects do not seem to play an important role. The continuum $(n \rightarrow \infty)$ limit of PRISM theory performs remarkably well, considering its simplicity and modest computational demands, provided the corresponding exact form factor (17) is used. The pressures obtained when the Lorentzian approximant (17) to the form factor is used in the PRISM calculations, are systematically too high. The substantial differences observed between the results of PRISM theory obtained with the exact and the Lorentzian form factor are reassuring, since the latter has also been used for the thread model of polymers, and leads to the prediction that the equation of state of thin, non-intersecting polymers of ra- dius of gyration $R_{\mathrm{g}}$ would be the same as that of infinitely thin platelets of radius $R=R_{\mathrm{g}}$.

We are considering a number of extensions of the present work. In order to investigate the isotropic-tonematic phase transition and other possible transitions to anisotropic phases, RISM and PRISM theories must be generalized to allow for anisotropic form factors, mirroring the orientation of platelets relative to a director. The present work will also be extended to binary mixtures of platelets of different diameters (and hence numbers of sites) and aspect ratios. This will introduce a competition between depletion-induced demixing and orientational ordering, which is expected to lead to rich phase behaviour [15, 18, 35]. A final extension of our work will be to charged platelets, modelled by using interaction sites which carry charges [19]. This extension is important for a description of aqueous dispersions of natural, or synthetic clays, like the widely studied Laponite, which exhibit poorly understood Coulombinduced gelling behaviour [36]. Our preliminary investigations [18] encourage us to pursue a more systematic investigation of charged platelets within the framework of RISM and PRISM theory.

\section{ACKNOWLEDGEMENTS}

DC wishes to thank Prof. C. Caccamo for his continuous encouragement and support during this work. Helpful discussions with Dr. R. Blaak are also gratefully acknowledged.
[1] L. Onsager, Phys. Rev. 62, 558 (1942); Ann. N.Y. Acad. Sci. 51, 627 (1949).

[2] For a review see S. Fraden in Observation, Prediction and Simulation of Phase Transitions in Complex Fluids, edited by M. Baus, L. F. Rull and J. P. Ryckaert (Kluwer, Dordrecht, 1994).

[3] I. Langmuir, J. Chem. Phys. 6, 873 (1938).

[4] F. M. van der Koij and H. N. W. Lekkerkerker, J. Phys. Chem. B 102, 7829 (1998).

[5] R. Eppenga and D. Frenkel, Molec. Phys. 52, 1303 (1984).

[6] H. Reiss, H. L. Frisch, and J. L. Lebowitz, J. Chem. Phys. 31, 369 (1959).

[7] For a review see M. A. Cotter in Molecular Physics of Liquid Crystals, edited by G. R. Luckhurst and G. W. Gray (Academic Press, London, 1979).

[8] B. M. Mulder and D. Frenkel, Molec. Phys. 55, 119 (1985).

[9] K. L. Savithramma and N. V. Madhusudana, Molec. Crys. Liq. Cryst. 74, 243 (1981).

[10] T. Boublik, J. Chem. Phys. 63, 4084 (1975).

[11] J. A. C. Veerman and D. Frenkel, Phys. Rev. A 45, 5632 (1992).

[12] S. D. Zhang, P. A. Reynolds, and J. S. Van Duijneveldt, Molec. Phys. 100, 3041 (2002).

[13] J. D. Parsons, Phys. Rev. A 19, 1225 (1979).

[14] S. D. Lee, J. Chem. Phys. 87, 4972 (1987); ibidem 89,
7036 (1989).

[15] H. H. Wensink, PhD dissertation, University of Utrecht (2004).

[16] Y. Rosenfeld, Phys. Rev. Lett. 63, 980 (1989); Y. Rosenfeld, D. Levesque, and J. J. Weis, J. Chem. Phys. 92, 6818 (1990).

[17] R. Zwanzig, J. Chem. Phys. 39, 1714 (1963).

[18] L. Harnau, D. G. Rowan, and J.-P. Hansen, J. Chem. Phys. 117, 11359 (2002).

[19] S. Kutter, J.-P. Hansen, M. Sprik, and E. Boek, J. Chem. Phys. 112, 311 (2000).

[20] Laporte Inorganics Laponite Technical Bulletin L104/90/A.

[21] D. Chandler and H. C. Andersen, J. Chem. Phys. 57, 1930 (1972); D. Chandler in Studies in Statistical Mechanics, vol. 8, 275, edited by J. L. Lebowitz and E. W. Montroll (North Holland, Amsterdam, 1982).

[22] For a review see P. A. Monson and G. P. Morriss, Adv. Chem. Phys. 77, 451 (1990).

[23] K. S. Schweizer and J. G. Curro, Phys. Rev. Lett. 58, 246 (1987); J. G. Curro and K. S. Schweizer, Macromolecules 20, 1928 (1987).

[24] For a review see K. S. Schweizer and J. G. Curro, Adv. Chem. Phys. 98, 1 (1997).

[25] L. Harnau, J.-P. Hansen, and D. Costa, Europhys. Lett. 53, 729 (2001).

[26] F. M. van der Koij, E. Kassapidou, and 
H. N. W. Lekkerkerker, Nature 406, 868 (2000); F. M. van der Koij, and H. N. W. Lekkerkerker, Phys. Rev. Lett. 84, 781 (2000); F. M. van der Koij, A. P. Philipse, and J. K. G. Dhont, Langmuir 16, 5317 (2000).

[27] F. O. Raineri and G. Stell, J. Phys. Chem. B 105, 11880 (2001).

[28] D. Chandler, R. Silbey, and B. Ladanyi, Molec. Phys. 46, 1335 (1982).

[29] J.-P. Hansen and I. R. McDonald, Theory of Simple Liquids, 2nd ed. (Academic Press, London, 1986).

[30] G. T. Pickett and K. S. Schweizer J. Chem. Phys. 112, 4881 (2000).

[31] L. Harnau and P. Reineker, J. Chem. Phys. 112, 437 (2000).
[32] A. Guinier and G. Fournet, Small Angle Scattering of $X$-rays (Wiley, New York, 1955).

[33] G. T. Pickett and K. S. Schweizer J. Chem. Phys. 110, 6597 (1999).

[34] See e.g. J. L. Barrat and J.-P. Hansen, Basic Concepts for Simple and Complex Liquids, p. 96 (Cambrisge University Press, 2003).

[35] H. H. Wensink, G. J. Vroege, and H. N. W. Lekkerkerker, J. Phys. Chem. B 105, 10610 (2001); H. H. Wensink and G. J. Vroege, Phys. Rev. E 65, 031716 (2002).

[36] A. Mourchid, A. Delville, J. Lambrad, E. Lécolier, and P. Levitz, Langmuir 11, 1942 (1995); H. Kellay, H. Tanaka, G. Wegdam, and J. Meunier, Langmuir 15, 7534 (1999). 